\title{
Astroglial Connexin43 Hemichannels Tune Basal Excitatory Synaptic Transmission
}

\author{
Oana Chever, ${ }^{*}$ Chun-Yao Lee, ${ }^{*}$ and Nathalie Rouach \\ Neuroglial Interactions in Cerebral Physiopathology, Center for Interdisciplinary Research in Biology, Collège de France, Centre National de la Recherche \\ Scientifique, Unité Mixte de Recherche 7241, Institut National de la Santé et de la Recherche Médicale U1050, 75005 Paris, France
}

Fast exchange of extracellular signals between neurons and astrocytes is crucial for synaptic function. Over the last few decades, different pathways of astroglial release of neuroactive substances have been proposed to modulate neurotransmission. However, their involvement in physiological conditions is highly debated. Connexins, the gap junction forming proteins, are highly expressed in astrocytes and have recently been shown to scale synaptic transmission and plasticity. Interestingly, in addition to gap junction channels, the most abundant connexin $(\mathrm{Cx})$ in astrocytes, $\mathrm{Cx} 43$, also forms hemichannels. While such channels are mostly active in pathological conditions, they have recently been shown to regulate cognitive function. However, whether astroglial $\mathrm{Cx} 43$ hemichannels are active in resting conditions and regulate basal synaptic transmission is unknown. Here we show that in basal conditions Cx43 forms functional hemichannels in astrocytes from mouse hippocampal slices. We furthermore demonstrate that the activity of astroglial $\mathrm{Cx} 43 \mathrm{hemichannels} \mathrm{in}$ resting states regulates basal excitatory synaptic transmission of hippocampal CA1 pyramidal cells through ATP signaling. These data reveal $\mathrm{Cx} 43$ hemichannels as a novel astroglial release pathway at play in basal conditions, which tunes the moment-to-moment glutamatergic synaptic transmission.

Key words: astrocytes; basal synaptic transmission; connexin43; hemichannel; hippocampus; physiology

\section{Introduction}

Astrocytes are active partners of neurons. They regulate neuronal activity through various mechanisms, including the release of neuroactive substances (Nedergaard and Verkhratsky, 2012; Araque et al., 2014). The dialog that occurs in physiological conditions and the nature of the various underlying molecular mechanisms of astroglial gliotransmitter release are still unclear. Indeed, different molecular pathways mediate the release of gliotransmitters such as vesicular exocytosis, connexin $(\mathrm{Cx})$ and pannexin (Px) hemichannels (HCs), P2X7, TREK-1, Best1, and swelling-activated anion channels. However, their involvement in basal conditions is a matter of intense debate and study (Ham-

Received Jan. 2, 2014; revised May 27, 2014; accepted June 27, 2014.

Author contributions: 0.C., C.-Y.L., and N.R. designed research; $0 . C$. ., C.Y.L., and N.R. performed research; $0 . C$., C.-Y.L., and N.R. analyzed data; $0 . C$. and N.R. wrote the paper.

This work was supported by grants from Agence Nationale de la Recherche (Programme Jeunes chercheurs and Programme Blanc Neurosciences), Fédération pour la Recherche sur le Cerveau, INSERM, and La Pitié Salpêtrière Hospital (translational research contract) to N.R.; and Fondation pour la Recherche Médicale postdoctoral fellowships to 0.C. and C.-Y.L. We thank P. Ezan, C. Escartin, G. Ghézali, J.P. Mothet, J. Teillon, and Y. Dupraz for technical assistance; G. Dallérac for helpful comments on the manuscript; and K. Willecke (University of Bonn, Bonn, Germany) for providing the $\mathrm{C} \times 43^{\mathrm{f} / \mathrm{fl}} \mathrm{h}$ hGFAP-Cre mice.

${ }^{*} 0$.C. and C.-Y.L. contributed equally to this work.

The authors declare no competing financial interests.

Correspondence should be addressed to Nathalie Rouach, Neuroglial Interactions in Cerebral Physiopathology, CIRB, Collège de France, CNRS UMR 7241, INSERM U1050, 11, Place Marcelin Berthelot, 75005 Paris, France. E-mail: nathalie.rouach@college-de-france.fr.

0 . Chever's present address: Institute of Molecular and Cellular Pharmacology, CNRS UMR 7275 and University of Nice-Sophia Antipolis, Valbonne, France.

DOI:10.1523/JNEUROSCI.0015-14.2014

Copyright $\odot 2014$ the authors $\quad 0270-6474 / 14 / 3411228-05 \$ 15.00 / 0$ ilton and Attwell, 2010; Panatier et al., 2011; Agulhon et al., 2012; Nedergaard and Verkhratsky, 2012).

Typically, astrocytes abundantly express the gap junction protein subunits $\mathrm{Cx} 43$ and $\mathrm{Cx} 30$, which mediate the formation of extensive astroglial networks (Pannasch and Rouach, 2013). Gap junction channels, formed by the apposition of two HCs belonging to adjacent cells, enable direct cytoplasmic exchange of numerous small molecules, such as energy metabolites, ions, or neurotransmitters (Pannasch and Rouach, 2013). However, in addition to the classical formation of gap junctions, hexamers of $\mathrm{Cx} 43$ are also inserted at astroglial membranes as large-pore single HCs, which mediate direct cytosolic exchange with the extracellular space (Giaume et al., 2013). Cx43 HCs can release various neuroactive substances such as potassium, ATP, or glutamate (Giaume et al., 2013), which are known to modulate neuronal activity. Until now, astroglial HCs have been shown to be active mostly in pathological conditions, such as inflammation (Giaume et al., 2013) or during high neuronal activity (Torres et al., 2012). Yet, astroglial Cx43 HC activity in resting conditions and its putative role in regulating basal synaptic transmission have never been investigated.

Astroglial Cx HCs are indeed usually assumed to be inactive in basal conditions, due to their low open probability at resting membrane potential and ionic concentrations (Contreras et al., 2003; Kang et al., 2008). Nonetheless, outside the brain, Cx HCs do open in physiological conditions, such as in the retina where they regulate neural progenitor proliferation (Pearson et al., 2005), or in the inner ear where they sustain long-range intercellular calcium signals (Anselmi et al., 2008), as shown in astrocytes (Giaume et al., 2013). Remarkably, it has also recently been 

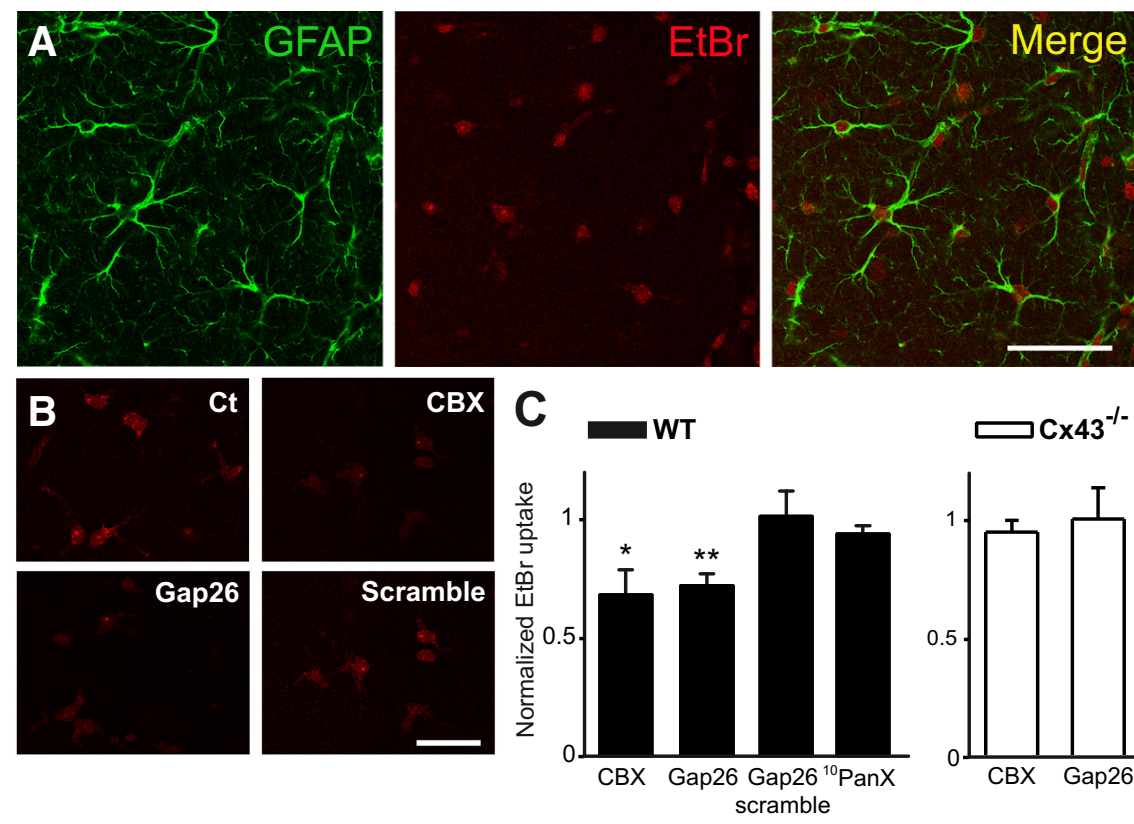

Figure 1. Astroglial $\mathrm{C} \times 43 \mathrm{HCs}$ are open in basal conditions. $A$, Representative basal EtBr uptake (red) in stratum radiatum astrocytes labeled with GFAP (green) in hippocampal slices. Scale bar, $50 \mu \mathrm{m}$. B. Higher magnification of astroglial EtBr uptake (red) in basal conditions ( $(\mathrm{t})$, and in the presence of (BX (200 $\mu \mathrm{M})$, Gap26 (100 $\mu \mathrm{M})$, or Gap26 scramble peptides (100 $\mu \mathrm{M})$, applied 15 min prior and during EtBr uptake assay. Scale bar, $25 \mu \mathrm{m}$. C, Astrocytic EtBr uptake normalized to control conditions in slices from wild-type mice (WT) treated with CBX (200 $\mu \mathrm{m}, n=7)$, Gap26 (100 $\mu \mathrm{m}, n=7)$, Gap26 scramble (100 $\mu \mathrm{m}, n=7)$ and ${ }^{10}$ Panx $(400 \mu \mathrm{m}, n=5)$ peptides (left), and from astroglial conditional CX43 knock-out mice $\left(\mathrm{CX}_{4} 3^{-1-}\right)$ treated with CBX (200 $\mu \mathrm{M}, n=$ 4) or Gap26 (100 $\mu \mathrm{m}, n=3$; right). Asterisks indicate statistical significance performed on raw data ( ${ }^{*} p<0.05,{ }^{* *} p<0.01$, Student's paired $t$ test).

shown that astroglial $\mathrm{Cx} 43 \mathrm{HCs}$ regulate cognitive function, since blocking such channels in the amygdala impairs fear memory consolidation (Stehberg et al., 2012). In addition, we have recently shown that the deletion of astroglial Cxs alters synaptic transmission and plasticity (Pannasch et al., 2011). Altogether, these data suggest that $\mathrm{Cx}$ HCs regulate synaptic function. We here show that hippocampal astrocytic $\mathrm{Cx} 43 \mathrm{HCs}$ are open under resting conditions and modulate basal synaptic transmission through ATP signaling.

\section{Materials and Methods}

Animals. Experiments were performed according to the guidelines of European Community Council Directives of January 1, 2013 (2010/63/ $\mathrm{EU}$ ), and all efforts were made to minimize the number of animals used and their suffering. Experiments were performed on the hippocampus of wild-type mice and $\mathrm{Cx} 43^{\mathrm{fl} / \mathrm{fl}}$ :hGFAP-Cre mice $\left(\mathrm{C} \times 43^{-/-}\right)$, with conditional deletion of $\mathrm{Cx} 43$ in astrocytes (Theis et al., 2003). For all analyses, mice of both genders and their littermates were used (PN16-PN25).

Acute hippocampal slices. Acute transverse hippocampal slices (300$400 \mu \mathrm{m}$ ) were prepared as previously described (Pannasch et al., 2011). Slices were maintained at room temperature in a storage chamber containing a standard artificial CSF [ACSF (in mM): $119 \mathrm{NaCl}, 2.5 \mathrm{KCl}, 2.5$ $\mathrm{CaCl}_{2}, 1.3 \mathrm{MgSO}_{4}, 1 \mathrm{NaH}_{2} \mathrm{PO}_{4}, 26.2 \mathrm{NaHCO}_{3}$, and 11 glucose, saturated with $95 \% \mathrm{O}_{2}$ and $5 \% \mathrm{CO}_{2}$ ] for at least $1.5 \mathrm{~h}$ before the experiments. Basal $\mathrm{HC}$ activity and synaptic transmission were analyzed at a depth of $40-80$ $\mu \mathrm{m}$ from the surface of acute slices and at a recovery time of at least 90 min after the slicing procedure, because in such conditions astrocytes exhibited no sign of reactivity, as assessed by GFAP and vimentin stainings. Indeed, in acute hippocampal slices fixed with $4 \%$ paraformaldehyde after a 90 min incubation period in oxygenated ACSF, we found that astrocytes did not show hallmarks of reactive gliosis, since they display: (1) no enhanced expression of GFAP, compared with sections from fixed brain following intracardiac perfusion of $4 \%$ paraformaldehyde (perfusion-fixed tissue; total GFAP staining area of individual astrocytes:

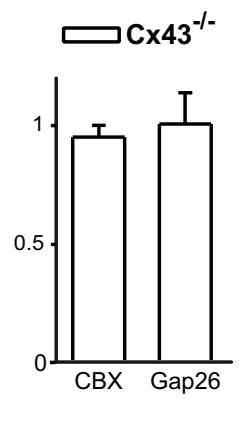

fixed acute slice, $197 \pm 16.33 \mu \mathrm{m}^{2}, n=41$; perfusion fixed tissue, $191 \pm 8.64 \mu \mathrm{m}^{2}, n=40$; $p>0.05$, unpaired $t$ test); and (2) no vimentin immunoreactivity (Pannasch et al., 2011). The recovery period after acute slice preparation is crucial for astroglial integrity because GFAP immunoreactivity was markedly reduced after a 90 min recovery period compared with a 10 min recovery period (relative astroglial GFAP intensity: 10 min recovery, $1.02 \pm 0.07, n=63$ cells; 90 min recovery, $0.47 \pm 0.03, n=82$ cells; $p<0.001$, unpaired $t$ test, Welch corrected).

Dye uptake by hemichannels. Living slices were distributed in small customized submerged chambers and incubated for $10 \mathrm{~min}$ in ACSF. To investigate the contribution of $\mathrm{Cx}$ and Px HCs to dye uptake, independent experiments were performed where slices were preincubated $15 \mathrm{~min}$ before and during the application of ethidium bromide (EtBr; 314 $\mathrm{Da}, 4 \mu \mathrm{M}, 10 \mathrm{~min}$ ), an HC-permeable fluorescent tracer, with carbenoxolone (CBX; 200 $\mu \mathrm{M})$, a Cx/Px HC blocker, Gap26 (100 $\mu \mathrm{M})$, a Cx43 HC blocking peptide, Gap26 scramble $(100 \mu \mathrm{M})$, a scramble Gap26 peptide, or ${ }^{10}$ Panx1 (400 $\left.\mu \mathrm{M}\right)$, a Px1 HC blocking peptide. Slices were then rinsed for $15 \mathrm{~min}$ in ACSF, fixed for $2 \mathrm{~h}$ in $4 \%$ paraformaldehyde, immunostained for GFAP (monoclonal mouse antiGFAP antibodies (1:500, clone GA-5; Sigma), with anti-mouse antibodies conjugated to $\mathrm{Al}-$ exa Fluor 488 (1:4000; Invitrogen) and mounted in Fluoromount. Labeled cells were examined in a confocal laser-scanning microscope (TCS SP5, Leica). Stacks of consecutive confocal images with high-bit depth color (16 bit) were taken at $1 \mu \mathrm{m}$ intervals and acquired in sequential mode with lasers (488 $\mathrm{nm}$ for GFAP and $561 \mathrm{~nm}$ for EtBr). Dye uptake analysis was performed in stratum radiatum CA1 astrocytes that were positive for GFAP. Fluorescence intensity was digitized in arbitrary units in 65,000 shades of gray with ImageJ software (National Institutes of Health). Dye uptake was evaluated and expressed as the difference between the fluorescence measured in astrocytes (20-40 cells per slice) and the background fluorescence measured in the same field where no labeled cells were detected.

Electrophysiology. Slices were transferred in a submerged recording chamber mounted on an Olympus BX51WI microscope equipped for infrared-differential interference contrast microscopy and were perfused with standard ACSF $(2 \mathrm{ml} / \mathrm{min}$, room temperature). All experiments were performed in CA1 stratum radiatum in the presence of picrotoxin $(100 \mu \mathrm{M})$, and a cut was made between CA3 and CA1 to prevent epileptiform bursts. Field EPSPs (fEPSPs) were recorded with glass pipettes (2-5 M $\Omega$ ) filled with ACSF. Whole-cell recordings were obtained from visually identified CA1 pyramidal cells using $5-8 \mathrm{M} \Omega$ glass pipettes filled with the following (in mM): 107.5 Cs-gluconate, 20 HEPES, 0.2 EGTA, 8 Na-gluconate, 8 TEA-Cl, 4 Mg-ATP, $0.3 \mathrm{Na}_{3}$-GTP, and 5 QX314, pH 7.4 (290 mOsm). Postsynaptic responses were evoked by Schaffer collateral single low stimulation $(10-20 \mu \mathrm{A}, 0.1 \mathrm{~ms}, 0.1 \mathrm{~Hz})$ through a glass pipette (2-5 M $\Omega$ ) filled with ACSF and located at a distance of $200 \mu \mathrm{m}$ from the recorded area. Recordings were acquired with Axopatch-1D amplifiers (Molecular Devices), digitized at $10 \mathrm{kHz}$, filtered at $2 \mathrm{kHz}$, stored, and analyzed on computer using pClamp9 and Clampfit10 software (Molecular Devices).

Bioluminescence imaging. ATP release was measured from hippocampal slices maintained in 24-well plates containing oxygenated ACSF (three slices per well with $500 \mu \mathrm{l}$ of ACSF) through the determination of extracellular ATP levels using the luciferin-luciferase assay (ATPLite kit, PerkinElmer) and a luminometer (Tristar LB941, Berthold). Each condition was run in triplicate, and absolute values were obtained from ATP standards. The release of extracellular ATP from hippocampal slices was 
also dynamically and locally imaged by chemiluminescence emitted from the reaction of luciferase and luciferin, delivered locally and continuously through a $5 \mathrm{M} \Omega$ glass pipette connected to a micropump. Photon production was captured by a high-sensitivity EM-CCD camera (iXon 897, Andor) with a $20 \times$ water-immersion objective (numerical aperture 0.95; Olympus) at $1 \mathrm{~Hz}$ with $5 \mathrm{~ms}$ exposure time using Axon Imaging Workbench software (Molecular Devices).

Statistics. All data are expressed as the mean \pm SEM, and $n$ represents the number of independent experiments, unless otherwise stated. Statistical significance was determined on raw data by paired or unpaired $t$ tests and one-way repeated-measures ANOVA followed by Dunnett or Bonferroni post-test.

Drugs. Gap26 (VCYDKSFPISHVR), Gap26 scramble (PSFDSRHCIVKYV), and ${ }^{10}$ Panx1 (WRQAAFVDSY) were synthesized by Thermo Fisher Scientific (purity, $>95 \%$ ), and all others products were from Sigma.

\section{Results}

Astroglial Cx43 hemichannels are active in basal conditions

To investigate whether astroglial HCs are functional in basal conditions, we performed EtBr uptake assay in stratum radiatum astrocytes from acute hippocampal slices. The majority of GFAPpositive astrocytes exhibited a basal EtdBr labeling in control conditions (Fig. 1A), which is currently thought to reflect nonspecific staining. However, we found that such basal astrocytic EtBr labeling is actually partly mediated by active uptake, because it was decreased by $\sim 32 \%$ when HCs were acutely inhibited by CBX (200 $\mu \mathrm{M}, 15 \mathrm{~min})$, a broad-spectrum blocker of both Cx and Px HCs $(-31.8 \pm 10.6 \%, n=7, p<0.05$; Fig. $1 B, C)$. HCs in astrocytes are formed by $\mathrm{Cx} 43$ and Pxl subunits (Giaume et al., 2013). Thus, to decipher the molecular identity of the HCs underlying basal uptake, we acutely and specifically blocked $\mathrm{Cx} 43$ or Px1 HCs using a short application (15 min) of extracellular loop mimetic peptides (Giaume et al., 2013). Gap26 (100 $\mu \mathrm{M})$, a specific $\mathrm{Cx} 43 \mathrm{HC}$ blocking peptide, significantly decreased astrocytic basal EtBr uptake $(-28.5 \pm 4.8 \%, n=7, p<0.01$; Fig. $1 B, C)$ to a similar extent as CBX, whereas the Gap26 scramble peptide $(100 \mu \mathrm{M})$ had no effect $(n=7, p>0.05$; Fig. $1 B, C)$. To further test the specific involvement of $\mathrm{Cx} 43 \mathrm{HCs}$, we used astroglial conditional $\mathrm{Cx} 43$ knock-out mice $\left(\mathrm{Cx} 43^{-1-}\right)$, in which astrocytes do not express Cx43, and found that Gap26, as well as CBX, had no significant effect on basal EtBr uptake in $\mathrm{Cx} 43^{-1-}$ astrocytes (CBX, $n=4$; Gap26, $n=3 ; p>0.05$; Fig. $1 C$ ). Finally, ${ }^{10} \mathrm{Panx}(400 \mu \mathrm{M})$, a specific Px1 HC mimetic peptide, also did not alter basal uptake in wild-type astrocytes $(n=5, p>0.05$, Fig. $1 C)$. Altogether, these results indicate that $\mathrm{Cx} 43 \mathrm{HCs}$, but not $\mathrm{Px} 1$ $\mathrm{HCs}$, are functional in astrocytes during resting conditions.

\section{Astroglial Cx43 hemichannels contribute to excitatory synaptic transmission through ATP signaling}

Astroglial $\mathrm{Cx} 43$ HCs release numerous neuroactive molecules (Giaume et al., 2013), which can modulate neuronal excitatory activity. We thus investigated whether the basal activity of $\mathrm{Cx} 43$ HCs that we identified regulates basal glutamatergic synaptic transmission. To do so, we performed whole-cell patch-clamp recordings of excitatory synaptic activity induced by single and low stimulation of Schaffer collaterals in CA1 pyramidal cells. We found that acute application of the Cx43 HC blocker Gap26 (100 $\mu \mathrm{M}$ ) rapidly induced (within $<5 \mathrm{~min}$ ) a $\sim 33 \%$ decrease in EPSC amplitude $(-33.2 \pm 3.4 \%, n=9, p<0.01$; Fig. $2 A, B)$, while the Gap26 scramble peptide $(100 \mu \mathrm{M}, 10 \mathrm{~min})$ had no significant effect ( $n=8, p>0.05$; Fig. $2 C)$. Furthermore, Gap26 (100 $\mu \mathrm{M}, 10$ $\mathrm{min}$ ) did not alter excitatory synaptic transmission in hippocampal slices from astroglial conditional $\mathrm{Cx} 43$ knock-out mice $\left(\mathrm{Cx} 43^{-/-}, n=17, p>0.05\right.$; Fig. $\left.2 D\right)$, thus demonstrating the
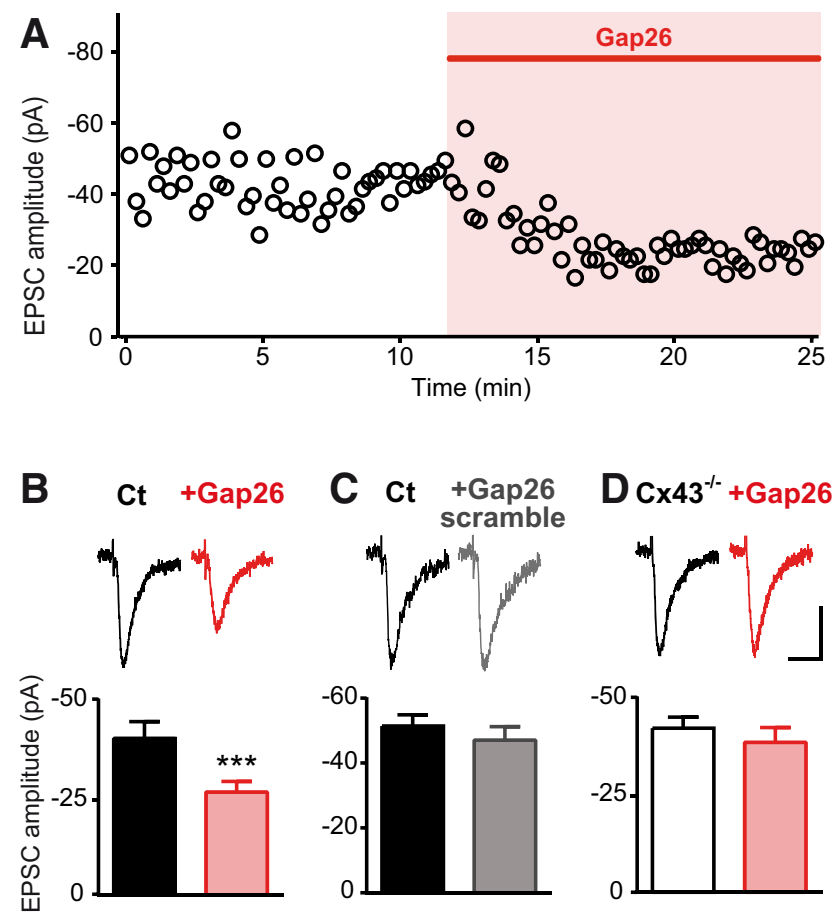

Figure 2. Astroglial Cx43 HCs modulate basal excitatory synaptic transmission. $\boldsymbol{A}, \boldsymbol{B}$, Astroglial Cx43 HCs potentiate synaptic transmission. A, Quantification over time of EPSC amplitudes recorded in a representative CA1 pyramidal cell before and during Gap26 application (red). $\boldsymbol{B}$, Sample traces and quantification of evoked EPSCs recorded in CA1 pyramidal neurons before [control (Ct), black] and during Gap26 application (Gap26, red, $n=9)$. C, D, Specificity of the Gap26 mimetic peptide. C, Sample traces and quantification of evoked EPSCs recorded in CA1 pyramidal cells before (black) and during (gray) the application of the Gap26 scramble peptide $(n=8)$. $\boldsymbol{D}$, Sample traces and quantification of evoked EPSCs recorded in CA1 pyramidal neurons from astroglial conditional Cx43 knockout mice ( $\left(x 43^{-1-}\right)$ before (black) and during (red) the application of Gap26 ( $\left.n=17\right)$. Gap26 and scramble peptides were applied at $100 \mu \mathrm{m}$, and their effects were quantified after 10 min. Calibration: 20 pA, 20 ms. Asterisks indicate statistical significance $\left({ }^{* *} p<\right.$ 0.001 , Student's paired $t$ test).

specific effect of the Gap26-mediated astroglial Cx43 HC inhibition on synaptic activity. Altogether, these data indicate that astroglial Cx43 HCs strengthen basal excitatory synaptic transmission.

Because ATP is a gliotransmitter known to permeate $\mathrm{Cx} 43$ HCs (Kang et al., 2008) and to increase excitatory synaptic transmission through P2 receptor activation (Pankratov et al., 1998; Gordon et al., 2005), we investigated the involvement of ATP signaling in the $\mathrm{Cx} 43 \mathrm{HC}$ regulation of basal synaptic activity. Using a luciferin-luciferase luminescence assay, we quantitatively measured with a luminometer ATP release from hippocampal slices bathed in a defined volume of ACSF, and found an approximately fivefold decrease in basal extracellular ATP concentration in Gap26-treated slices $(n=4, p<0.001$; Fig. $3 A)$. In addition, using real-time detection of local extracellular ATP variations in hippocampal slices, we found that Gap26 readily (within $2 \mathrm{~min}$ ) decreased ATP levels $(n=7, p<0.01$; Fig. $3 B)$. Altogether, these data show that ATP is tonically released through $\mathrm{Cx} 43 \mathrm{HCs}$ in resting conditions. To investigate whether basal extracellular ATP exerts a tonic facilitation of excitatory neurotransmission by the direct activation of ATP purinergic receptors, we acutely inhibited $\mathrm{P} 2$ receptors using the broad-spectrum antagonists $\mathrm{RB} 2$ and PPADS $(30 \mu \mathrm{M}, 20 \mathrm{~min})$, and found that this decreased excitatory synaptic transmission by $\sim 31 \%(-31.1 \pm 3.8 \%, n=5$, $p<0.01$; Fig. $4 A$ ), as observed with Gap26 inhibition of $\mathrm{Cx} 43$ HCs. In addition, in Gap26-treated slices (100 $\mu \mathrm{M}, 10 \mathrm{~min}$ ), nor- 

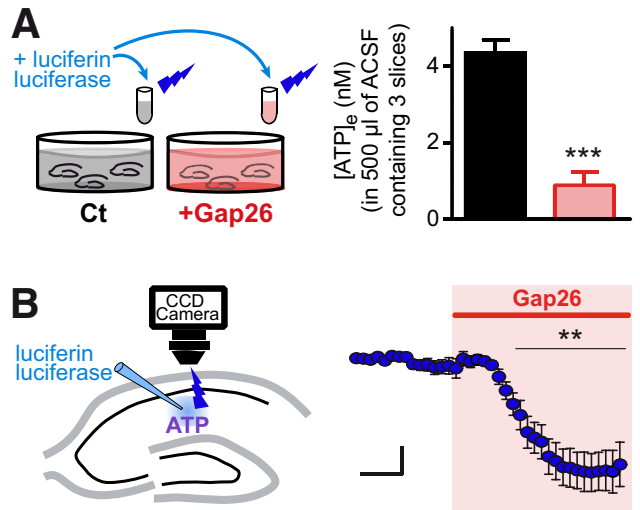

Figure 3. Astroglial $\mathrm{C} 43 \mathrm{HCs}$ release ATP in basal conditions. $A$, Left, Schematic representation of the experimental design to measure quantitatively ATP release from hippocampal slices maintained in wells with oxygenated ACSF. Right, Quantification of luminescence detection of ATP release measured in $500 \mu \mathrm{l}$ of ACSF containing three hippocampal slices in control conditions (Ct; black) and after Gap26 application (30 min; red; $n=4)$. B, Left, Schematic representation of dynamic extracellular ATP imaging in a hippocampal slice, using local delivery of luciferin-luciferase through a pipette located in CA1 stratum radiatum. Right, Quantification over time of luminescence detection of extracellular ATP levels before and during Gap26 application (red, $n=7$ ). Calibration: 1 arbitrary unit, 1 min. Asterisks indicate statistical significance [Student's paired $t$ test $(\boldsymbol{A})$ or one-way repeated-measures ANOVA and Dunnett's post hoc test $\left.(\boldsymbol{B}):{ }^{* *} p<0.01,{ }^{* * *} p<0.001\right]$.

mal excitatory transmission was rescued by local application of ATP $(1 \mu \mathrm{M}, 3$ min applied with a $250-\mu \mathrm{m}$-diameter barrel at the slice surface; fEPSP slope before and during the application of Gap26 alone or combined with ATP: control, $-0.18 \pm 0.01 \mathrm{mV} /$ $\mathrm{ms}$; Gap26, $-0.14 \pm 0.01 \mathrm{mV} / \mathrm{ms}$; difference: $-24.6 \pm 1.1 \%$ compared with control, $p<0.001$; Gap26 plus ATP, $-0.17 \pm$ $0.01,+24 \pm 7.1 \%$ compared with Gap26, $n=7, p<0.01$, repeated-measures ANOVA with Bonferroni post-test). Finally, when hippocampal slices were pretreated with ATP P2 receptor antagonists (RB2 and PPADS, $30 \mu \mathrm{M}, 20 \mathrm{~min}$ ), acute inhibition of Cx43 HCs by Gap26 failed to induce the decrease in EPSC amplitude $(n=6, p>0.05$; Fig. $4 B, C)$, which was previously observed under control conditions (Fig. 2A,B). Thus, the inhibition of ATP P2 receptors occludes the effect of $\mathrm{Cx} 43 \mathrm{HC}$ blockers on synaptic transmission.

Altogether, these data indicate that astroglial $\mathrm{Cx} 43 \mathrm{HCs}$ contribute to basal excitatory synaptic transmission via ATP signaling.

\section{Discussion}

The present results reveal astroglial $\mathrm{Cx} 43 \mathrm{HCs}$ as a novel pathway mediating signal exchanges between astrocytes and neurons in basal conditions. This result is remarkable since Cx or Px HCs have thus far been assumed to be active mostly in pathological conditions, such as ischemia, inflammation, or Alzheimer's disease (Giaume et al., 2013).

Acute slice preparation, although extensively used in neurophysiological investigations, is an experimental model that does not fully reflect the physiological conditions of an intact brain. In particular, early stages of reactive gliosis (Takano et al., 2014) and transient loss of glycogen granules (Fiala et al., 2003) have been reported in astrocytes from acute hippocampal slices, raising the possibility that traumatic injury during the slicing procedure might induce $\mathrm{Cx} 43 \mathrm{HCs}$ activation. We, however, found that at a depth of $40-80 \mu \mathrm{m}$ from the acute slice surface, and at a recovery time of at least $90 \mathrm{~min}$ after the slicing procedure (i.e., the conditions used in our experiments), astrocytes exhibit no sign of reactivity (see Materials and Methods). In addition, the contri-
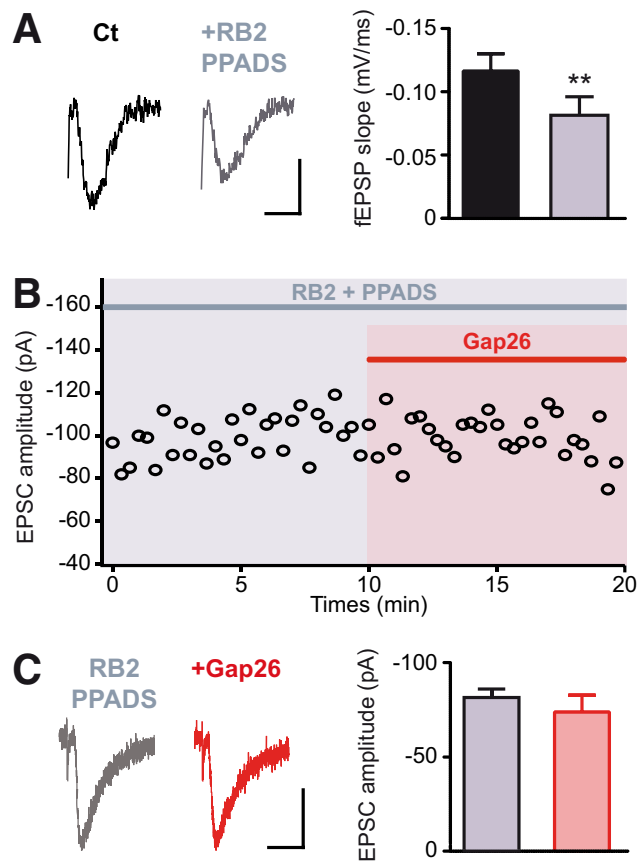

Figure 4. ATP signaling mediates astrocytic $\mathrm{C} \times 43 \mathrm{HC}$ regulation of synaptic transmission. $\boldsymbol{A}$ Endogenous extracellular ATP increases basal excitatory synaptic transmission through P2 receptors. Sample traces and quantification of evoked CA1 fEPSPs recorded before ( $\mathrm{Ct}$; black) and during application of ATP P2 receptor antagonists (RB2 + PPADS; gray, $n=5$ ). Calibration: 0.2 $\mathrm{mV}, 10 \mathrm{~ms} . \boldsymbol{B}, \boldsymbol{C}$, Blockade of ATP P2 receptors occludes the effect of $\mathrm{Cx} 43 \mathrm{HC}$ inhibition on synaptic transmission. $\boldsymbol{B}$, Quantification over time of EPSC amplitudes recorded in a representative CA1 pyramidal cell in the presence of ATP P2 receptor antagonists (RB2+PPADS preincubation; gray) before and during Gap26 application (red). C, Corresponding sample traces and quantification of averaged EPSC amplitudes $(n=6)$. Calibration: $40 \mathrm{pA}, 40 \mathrm{~ms}$. The effect of Gap26 $(100 \mu \mathrm{M})$ and a mix of RB2 and PPADS $(30 \mu \mathrm{M})$ was quantified after a 10-20 min application. Asterisks indicate statistical significance ${ }^{* *} p<0.01$, Student's paired $t$ test).

bution of $\mathrm{Cx}$ HCs to physiological processes has recently been reported in vivo in the brain (Stehberg et al., 2012), suggesting that the $\mathrm{Cx} 43 \mathrm{HC}$ activity we report here under basal conditions in hippocampal slices may indeed occur in intact physiological conditions.

Notably, we found that HCs composed of $\mathrm{Cx} 43$, but not Px1, contribute specifically to the basal uptake of $\mathrm{EtBr}$ in astrocytes. To reveal in acute slices such significant $\mathrm{Cx} 43$-mediated HC uptake in basal conditions, we used confocal imaging of astrocytes with high-bit depth color (16 bit), enabling reliable detection with high sensitivity of variations in intracellular fluorescence intensity. Interestingly, although the direct opening of HCs in basal conditions has not been directly investigated in recent studies proposing an involvement of astroglial Cx43 HCs in physiological processes (Stehberg et al., 2012; Torres et al., 2012), the activation of $\mathrm{Cx} 43 \mathrm{HCs}$, shown with dye uptake assays, has already been detected at resting membrane potential in HeLatransfected cells (Contreras et al., 2003). One can hypothesize that synaptic activity triggers basal activation of astroglial $\mathrm{Cx} 43$ HCs. Indeed, glutamate decrease in extracellular calcium or astroglial depolarization, which occur during neuronal activity, dynamically modulate HC activation (Orellana et al., 2013). Because Cx43 HCs may be located in the vicinity of synapses, local changes in extracellular ion or neurotransmitter levels at the tripartite synapse may induce selective and prominent activation of $\mathrm{Cx} 43 \mathrm{HCs}$ from perisynaptic astroglial processes.

Furthermore, we demonstrate that astroglial $\mathrm{Cx} 43 \mathrm{HC}$ activity in the resting state boosts the moment-to-moment basal gluta- 
matergic synaptic activity of CA1 pyramidal cells. Pharmacological inhibition of $\mathrm{Cx} 43 \mathrm{HCs}$ by mimetic peptides indeed acutely decreases the amplitude of EPSCs, while such blockers have no effect on astroglial conditional Cx43 knock-out mice. Our present findings nicely complement recent studies showing $\mathrm{Cx} 43 \mathrm{HC}$ contribution to neurophysiology. Indeed, a recent pioneering study has revealed a critical role for $\mathrm{Cx} 43 \mathrm{HCs}$ in physiological cognitive process by showing that intracerebral microinfusion of Cx43 HC blockers in the amygdala alters fear memory consolidation (Stehberg et al., 2012). Interestingly, in this study gliotransmitters, including ATP, were suggested to underlie such regulation. In addition, it has also recently been proposed that Cx43 HC activation by astroglial calcium waves, triggered by glutamate or calcium chelator uncaging, which mediate the decrease in extracellular calcium thought to occur during high neuronal activity, boosts interneuron excitability, and thereby inhibitory transmission, through ATP release targeting P2Y1 receptors on interneurons (Torres et al., 2012). This pathway has been suggested to provide a negative-feedback mechanism to tone down strong glutamatergic transmission and hyperexcitability of neuronal networks. Remarkably, in our study, the mechanism by which astroglial $\mathrm{Cx} 43 \mathrm{HCs}$ strengthen basal excitatory synaptic transmission also involves ATP release and activation of $\mathrm{P} 2$ purinergic receptors. However, we show here, using a short application of $\mathrm{Cx} 43 \mathrm{HC}$ blocking peptides, that $\mathrm{Cx} 43 \mathrm{HCs}$ acutely regulate basal neurotransmission mediated by excitatory synapses of CA1 pyramidal cells, while Torres et al. (2012), by using astroglial Cx43 transgenic mice, identified an effect on network activity involving interneurons during a high-activity regime. Because astroglial $\mathrm{Cx} 43 \mathrm{HCs}$ are not activated by extracellular ATP targeting P2 receptors (Iglesias et al., 2009; Orellana et al., 2013) and inhibitory synaptic activity was blocked by picrotoxin in our experiments to study specifically excitatory transmission, our data suggest that ATP released through astroglial Cx43 HCs directly activates $\mathrm{P} 2$ purinergic receptors on CA1 pyramidal cells, which are known to potentiate glutamatergic synaptic transmission (Pankratov et al., 1998; Gordon et al., 2005).

The regulation of basal excitatory synaptic transmission that we demonstrate here may well contribute to the effect of astroglial Cx43 HCs on memory consolidation (Stehberg et al., 2012). Additionally, one can hypothesize that the effects of astroglial Cx43 on physiological locomotor and exploratory activity in novel environments (Frisch et al., 2003; Theis et al., 2003), as well as on whisker-related sensory functions and plasticity (Han et al., 2014), may not only depend on Cx43-mediated gap junction coupling, but might also involve functional Cx43 HCs.

Thus, Cx43 HCs, a classic ATP release pathway in astrocytes that is usually thought to be at play in pathological situations, is actually also likely to be a key player in physiology by strengthening the moment-to-moment basal synaptic transmission.

\section{References}

Agulhon C, Sun MY, Murphy T, Myers T, Lauderdale K, Fiacco TA (2012) Calcium signaling and gliotransmission in normal vs. reactive astrocytes. Front Pharmacol 3:139. CrossRef Medline

Anselmi F, Hernandez VH, Crispino G, Seydel A, Ortolano S, Roper SD, Kessaris N, Richardson W, Rickheit G, Filippov MA, Monyer H, Mammano F (2008) ATP release through connexin hemichannels and gap junction transfer of second messengers propagate $\mathrm{Ca} 2+$ signals across the inner ear. Proc Natl Acad Sci U S A 105:18770-18775. CrossRef Medline Araque A, Carmignoto G, Haydon PG, Oliet SH, Robitaille R, Volterra A
(2014) Gliotransmitters travel in time and space. Neuron 81:728-739. CrossRef Medline

Contreras JE, Sáez JC, Bukauskas FF, Bennett MV (2003) Gating and regulation of connexin 43 (Cx43) hemichannels. Proc Natl Acad Sci U S A 100:11388-11393. CrossRef Medline

Fiala JC, Kirov SA, Feinberg MD, Petrak LJ, George P, Goddard CA, Harris KM (2003) Timing of neuronal and glial ultrastructure disruption during brain slice preparation and recovery in vitro. J Comp Neurol 465:90103. CrossRef Medline

Frisch C, Theis M, De Souza Silva MA, Dere E, Söhl G, Teubner B, Namestkova K, Willecke K, Huston JP (2003) Mice with astrocyte-directed inactivation of connexin43 exhibit increased exploratory behaviour, impaired motor capacities, and changes in brain acetylcholine levels. Eur J Neurosci 18:2313-2318. CrossRef Medline

Giaume C, Leybaert L, Naus CC, Sáez JC (2013) Connexin and pannexin hemichannels in brain glial cells: properties, pharmacology, and roles. Front Pharmacol 4:88. CrossRef Medline

Gordon GR, Baimoukhametova DV, Hewitt SA, Rajapaksha WR, Fisher TE, Bains JS (2005) Norepinephrine triggers release of glial ATP to increase postsynaptic efficacy. Nat Neurosci 8:1078-1086. CrossRef Medline

Hamilton NB, Attwell D (2010) Do astrocytes really exocytose neurotransmitters? Nat Rev Neurosci 11:227-238. CrossRef Medline

Han Y, Yu HX, Sun ML, Wang Y, Xi W, Yu YQ (2014) Astrocyte-restricted disruption of connexin-43 impairs neuronal plasticity in mouse barrel cortex. Eur J Neurosci 39:35-45. CrossRef Medline

Iglesias R, Dahl G, Qiu F, Spray DC, Scemes E (2009) Pannexin 1: the molecular substrate of astrocyte "hemichannels." J Neurosci 29:7092-7097. CrossRef Medline

Kang J, Kang N, Lovatt D, Torres A, Zhao Z, Lin J, Nedergaard M (2008) Connexin 43 hemichannels are permeable to ATP. J Neurosci 28:47024711. CrossRef Medline

Nedergaard M, Verkhratsky A (2012) Artifact versus reality-how astrocytes contribute to synaptic events. Glia 60:1013-1023. CrossRef Medline

Orellana JA, Martinez AD, Retamal MA (2013) Gap junction channels and hemichannels in the CNS: Regulation by signaling molecules. Neuropharmacology 75:567-582. CrossRef Medline

Panatier A, Vallée J, Haber M, Murai KK, Lacaille JC, Robitaille R (2011) Astrocytes are endogenous regulators of basal transmission at central synapses. Cell 146:785-798. CrossRef Medline

Pankratov Y, Castro E, Miras-Portugal MT, Krishtal O (1998) A purinergic component of the excitatory postsynaptic current mediated by $\mathrm{P} 2 \mathrm{X}$ receptors in the CA1 neurons of the rat hippocampus. Eur J Neurosci 10: 3898-3902. CrossRef Medline

Pannasch U, Rouach N (2013) Emerging role for astroglial networks in information processing: from synapse to behavior. Trends Neurosci 36: 405-417. CrossRef Medline

Pannasch U, Vargová L, Reingruber J, Ezan P, Holcman D, Giaume C, Syková E, Rouach N (2011) Astroglial networks scale synaptic activity and plasticity. Proc Natl Acad Sci U S A 108:8467-8472. CrossRef Medline

Pearson RA, Dale N, Llaudet E, Mobbs P (2005) ATP released via gap junction hemichannels from the pigment epithelium regulates neural retinal progenitor proliferation. Neuron 46:731-744. CrossRef Medline

Stehberg J, Moraga-Amaro R, Salazar C, Becerra A, Echeverría C, Orellana JA, Bultynck G, Ponsaerts R, Leybaert L, Simon F, Sáez JC, Retamal MA (2012) Release of gliotransmitters through astroglial connexin 43 hemichannels is necessary for fear memory consolidation in the basolateral amygdala. FASEB J 26:3649-3657. CrossRef Medline

Takano T, He W, Han X, Wang F, Xu Q, Wang X, Oberheim Bush NA, Cruz N, Dienel GA, Nedergaard M (2014) Rapid manifestation of reactive astrogliosis in acute hippocampal brain slices. Glia 62:78-95. CrossRef Medline

Theis M, Jauch R, Zhuo L, Speidel D, Wallraff A, Döring B, Frisch C, Söhl G, Teubner B, Euwens C, Huston J, Steinhäuser C, Messing A, Heinemann U, Willecke K (2003) Accelerated hippocampal spreading depression and enhanced locomotory activity in mice with astrocyte-directed inactivation of connexin43. J Neurosci 23:766-776. Medline

Torres A, Wang F, Xu Q, Fujita T, Dobrowolski R, Willecke K, Takano T, Nedergaard M (2012) Extracellular $\mathrm{Ca}(2)(+)$ acts as a mediator of communication from neurons to glia. Sci Signal 5:ra8. CrossRef Medline 